\title{
Morbi-mortalidade de crianças por causas externas, Distrito Federal, 2015-2019: estudo de correlação
}

\author{
Child morbidity and mortality by external causes, Distrito Federal, 2015-2019: correlation study \\ Morbilidad y mortalidad de niños por causas externas, Distrito Federal, 2015-2019: estudio de \\ correlación
}

Recebido: 26/07/2021 | Revisado: 31/07/2021 | Aceito: 03/08/2021 | Publicado: 08/08/2021

\author{
Luana Carolina Pinheiro do Valle \\ ORCID: https://orcid.org/0000-0002-3828-1042 \\ Universidade de Brasília, Brasil \\ E-mail: luanavalle.unb@gmail.com \\ Rita de Cássia Melão de Morais \\ ORCID: https://orcid.org/0000-0001-8526-0642 \\ Universidade de Brasília, Brasil \\ E-mail: ritamelao@gmail.com \\ Thyago José Arruda Pacheco \\ ORCID: https://orcid.org/0000-0002-8090-0644 \\ Universidade de Brasília, Brasil \\ E-mail: thyagojap@gmail.com
}

\begin{abstract}
Resumo
Objetivo: Analisar a correlação das internações hospitalares e óbitos pediátricos por causas externas com faixas etárias, sexo, mês e ano de ocorrência, no Distrito Federal de 2015 a 2019. Método: Estudo ecológico com análise de dados secundários, das causas externas do Sistema de Informações Hospitalares e Mortalidade, do DATASUS, na faixa etária de um a nove anos. Resultados: No período analisado ocorreram 6597 internações e 147 óbitos no Distrito Federal na faixa etária estudada. As crianças de um a quatro anos apresentaram maiores riscos de sofrerem envenenamentos, sufocamentos e afogamentos, enquanto de cinco a nove anos maiores riscos de sofrerem acidentes de transporte. As quedas constituíram a maior parte das internações; e afogamentos e acidentes de transporte maiores causas de óbitos. O sexo masculino foi o mais susceptível a sofrer quaisquer incidentes $(p<0,001)$. Os resultados foram inconclusivos acerca da raça/cor e análise dos meses não revelou maiores riscos $(\mathrm{p}=0,6643)$. Conclusões: Os acidentes nas duas faixas etárias apresentam perfis bem distintos. A maioria das crianças acidentadas continua pertencendo ao sexo masculino. O perigo dos afogamentos, atropelamentos e acidentes de trânsito resultarem mais frequentemente em óbitos enfatiza a gravidade desses incidentes, e, portanto, a maior necessidade de prevenção destes.
\end{abstract} Palavras-chave: Morbidade; Mortalidade da criança; Causas externas; Correlação de dados; Prevenção de acidentes.

\begin{abstract}
Objective: Analyze the correlation between hospitalization and child deaths by external causes and ages, genders, years and period in which they occurred, in Distrito Federal, from 2015 to 2019. Method: Data Correlation of the DATASUS's Hospital Information and Mortality System by external causes, in the age group from one to nine years. Results: There were 6597 hospitalizations and 147 deaths in the age group and period analyzed. Children from one to four years old had a higher risk of suffering poisoning, suffocation and drowning, while those from five to nine years old had greater risk of suffering transport accidents. Falls constituted most of the hospitalizations; drowning and transport accidents are the biggest causes of death. Boys most likely to suffer any incident. The results were inconclusive about the skin color and analysis of the months didn't reveal greater risks. Conclusions: Accidents in the two age groups have very different profiles. Most injured children are still male. The danger of drowning, being run over and traffic accidents more often resulting in deaths emphasizes the seriousness of these incidents and, therefore, the greater need for their prevention.
\end{abstract}

Keywords: Morbidity; Child mortality; Accidental injuries; Data correlation; Accident prevention.

\section{Resumen}

Objetivo: Evaluar la correlacion entre hospitalizaciones y muertes pediátricas por causas externas y edad, sexo, año y período de ocurrencia, en Distrito Federal, de 2015 a 2019. Método: Correlación de datos del Sistema de Información de Hospitalización y Mortalidad del DATASUS por causas externas, en niños de uno a nueve años. Resultados: Ocurrieron 6597 hospitalizaciones y 147 muertes en el grupo de edad y período analizados. Niños de uno a quatro años tuvieron mayor riesgo de sufrir intoxicaciones, asfixia y ahogamiento, mientras que los de cinco a nueve años de sufrir accidentes de transporte. Las caídas constituyeron la mayoría de las hospitalizaciones, mientras que ahogamientos y accidentes de transporte las de muerte. Los chicos tuvieron mayores probabilidades de sufrir algún incidente. Los resultados no fueron concluyentes sobre el color de la piel y el análisis de 
los meses no reveló mayores riesgos. Conclusiones: Los acidentes en los dos grupos de edad tienen perfiles bastante distintos. La mayoría de los niños heridos siguen siendo varones. El peligro de ahogamiento, atropello y los accidentes de tráfico com mayor frecuencia de fallecimiento enfatizan la gravedad de estos incidentes y, por tanto, la mayor necesidad de su prevención. Palabras clave: Morbilidad; Mortalidad del niño; Causas externas; Correlación de datos; Prevención de accidentes.

\section{Introdução}

A classificação de causas externas, segundo a Organização Mundial da Saúde (1975) inclui os acidentes, as violências propriamente ditas - auto e hetero infligidas (suicídio e homicídio/intervenção legal, respectivamente), complicações de assistência médica e cirúrgica; e caracteriza-os como eventos súbitos e inesperados. A Classificação Estatística Internacional de Doenças e Problemas Relacionados com a Saúde (CID-10) traz no capítulo XX essas causas externas de morbidade e mortalidade. Dentre o agrupamento denominado Acidentes estão os acidentes de transporte e outras causas externas de traumatismos acidentais (como por exemplo, quedas; afogamento e submersão acidentais; e envenenamento acidental por exposição a substâncias nocivas). Apesar do carácter definido como inesperado trazido pela classificação da OMS, os acidentes na maior parte das vezes são previsíveis, preveníveis e controláveis (Khazaei et al., 2016; Sleet, 2018)' principalmente ao se tratar da população pediátrica. Isso, porque, tendo em vista que o domicílio é o local com ocorrência mais frequente desses agravos (Ehrhardt, 2017; Malta et al., 2016; Ministério da Saúde, 2015), por ser onde as crianças passam a maior parte do tempo, supõese estarem sob constante vigilância.

O desenvolvimento infantil tem grande relevância para o entendimento da etiologia dos acidentes dessa faixa etária e influência no desenvolvimento de políticas públicas de saúde voltadas para a prevenção desses acidentes. Isso, porque, além de particularidades anatômicas, as crianças menores de cinco anos apresentam ainda uma imaturidade cognitiva, que se traduz principalmente na incapacidade para prever e evitar situações de perigo. Associadas à essa imaturidade estão a curiosidade, a inexperiência, a falta de noção corporal e de espaço, a falta de coordenação motora, a tendência a imitar comportamentos adultos, bem como as características da personalidade (hiperatividade, agressividade, impulsividade e distração) que somadas predispõem essas crianças à se exporem às situações de risco e, portanto, terem chances aumentadas de sofrerem acidentes (Brito et al., 2017; Gurgel \& Monteiro, 2016; Schwebel, 2019). De forma geral, por esses motivos, crianças menores de cinco anos estão mais propensas a sofrerem afogamentos, obstruções de vias aéreas e envenenamentos. À medida que vão crescendo e desenvolvendo, portanto, outras habilidades como a marcha e maior independência, além do fato de nessa idade geralmente começarem a vida escolar, as crianças maiores de cinco anos se expõe à diferentes riscos, em especial o de sofrerem acidentes de transporte, tanto na condição de pedestres como ao serem transportadas (Chang et al., 2018; Mackay \& Ryan, 2018).

Apenas no ano de 2020, na faixa etária de um a nove anos, no Brasil foram registradas 46198 internações hospitalares por causas externas que resultaram em 179 óbitos e no Distrito Federal 1632 internações que resultaram em onze óbitos (DATASUS). A despeito dos números parecerem pequenos, os acidentes na infância são de grande relevância, pois repercutem na criança, na família e na sociedade e gera custos sociais, econômicos e emocionais altos (Mackay \& Ryan, 2018; Mitchell et al., 2018) tendo em vista que esses acontecimentos também são responsáveis por traumatismos não fatais que se traduzem em diferentes tipos de deficiências à longo prazo (Goniewicz et al., 2018; Saadati et al., 2020).

As causas externas ocorridas na infância, portanto, devem ser consideradas como um problema de saúde pública importante. A identificação dessas causas externas e seus fatores associados servem como base para o fortalecimento das políticas públicas voltadas para a prevenção desses acidentes, e tem sido usada para o engajamento dos responsáveis e conscientização de seus papéis e do desenvolvimento de ambientes mais seguros para as crianças (Malta et al., 2016; Olsen el al., 2018; Zonfrillo et al., 2018). Apesar disso, na literatura existem poucos estudos que apresentam possíveis fatores causais para a ocorrência destes incidentes, bem como a correlação destes com o desenvolvimento infantil, em especial no Distrito Federal. A maior parte dos artigos com essa temática enfatiza a violência contra a criança. Dessa maneira, ainda são pouco explorados os aspectos que 
envolvem essas causas externas - excluídas violências- na pediatria, e, portanto, quem são essas crianças acidentadas e quais as causas dos óbitos nessa população.

\subsection{Objetivo}

Analisar a correlação entre as internações hospitalares e os óbitos pediátricos por causas externas na faixa etária de um a nove anos, sexo, cor, ano e período em que ocorreram, no Distrito Federal, Brasil, de 2015 a 2019.

\section{Metodologia}

\subsection{Aspectos éticos}

O estudo foi realizado em consonância com a Resolução do Conselho Nacional de Saúde nº 466 (2012, 12 de dezembro). Considerando que todos os dados coletados para esta pesquisa são de acesso irrestrito e disponível em domínio público e que as bases consultadas não continham informações sigilosas, foi dispensada a apreciação do projeto por um comitê de ética em pesquisa.

\subsection{Desenho, período e local de estudo}

Trata-se de um estudo ecológico retrospectivo que utilizou bancos de dados públicos para filtrar dados secundários sobre morbidade hospitalar e mortalidade do Sistema Único de Saúde (SUS). Estudos ecológicos são aqueles nos quais os dados analisados se referem a agregados de indivíduos, pertencentes à uma área delimitada (populações de países, regiões ou municípios, por exemplo). Busca-se encontrar nesse tipo de estudo uma associação entre exposições desses agregados e doenças ou condições relacionadas à saúde (Lima-Costa \& Barreto, 2003).

Para a coleta de dados foram pesquisados os seguintes bancos: Sistema de Informações Hospitalares do SUS (SIH/SUS) e Sistema de Informações sobre Mortalidade (SIM) com todos dados disponibilizados pelo DATASUS pelo sistema Tabwin. Os dados referentes à população pediátrica na faixa etária estudada, por anos, utilizada para o cálculo dos riscos relativos e razão de risco foram extraídos da base de dados da Companhia de Planejamento do Distrito Federal (CODEPLAN- DF, 2018).

O local objeto deste estudo foi o Distrito Federal, localizado na região Centro-Oeste do Brasil. Segundo a CODEPLANDF (2020) apresenta uma extensão de $5.779 \mathrm{~km}^{2}$ e possui 33 divisões denominadas regiões administrativas (Ras) tendo em vista que é a única unidade da Federação que não possui municípios. Possuía no último censo, de 2010, 2.570 .160 habitantes (CODEPLAN-DF, 2020) e, segundo a Pesquisa Distrital por Amostra de Domicílios (PDAD) 2018 tinha, neste ano, 458.273 crianças o que corresponde a $16 \%$ da população total do DF (CODEPLAN- DF, 2018). Esse grupo é composto por $49 \%$ de meninas e 51\% de meninos; e a distribuição por raça/cor é de 54\% de crianças negras e $46 \%$ não-negras no DF (CODEPLANDF, 2018).

\subsection{População ou amostra, critérios de inclusão e exclusão}

Foram coletados, no SIH/SUS, os dados referentes às internações hospitalares por causas externas no Distrito Federal, de setembro de 2014 à dezembro de 2019. Foram excluídas as internações como consequência de violência, tanto auto como hetero-infringidas, bem como complicações de assistência médica e cirúrgica. Dessa maneira a variável de critério foi composta pelas internações que tem como causa Acidentes de Transportes (V0-V99), Demais Causas Externas de Traumatismos Acidentais (W0- W99 e X0- X50), Causas de Intenção Indeterminada (Y10- Y34) e demais códigos de V01-Y98, que não os excluídos. As variáveis preditoras selecionadas foram: as faixas etárias de zero a quatro anos e de cinco a nove anos, sexo, cor/raça, mês e ano em que ocorreram. No SIM, foram coletados os dados de todos os óbitos ocorridos no período de 2015 a 2019 seguindo os mesmos critérios de seleção dos dados coletados no SIH/SUS. 
Todos os dados coletados foram exportados para uma planilha no programa Microsoft Excel 2010 e analisados no software Graph Pad Prism, adotando teste de Qui Quadrado e grau de confiabilidade de 95\% ( $\mathrm{p}<0,05)$ e medida de tendência por meio de teste de regressão não linear.

\section{Resultados}

\subsection{Morbidade Hospitalar}

No período de estudo ocorreram 6.597 internações hospitalares por causas externas em crianças na faixa etária de um a nove anos no Distrito Federal (DF), que custaram para o Estado um total de R \$ 82.971.250,29. Das 69.086 internações hospitalares no DF nessa faixa etária, o conjunto de causas externas, corresponde ao $3^{\text {a }}$ maior fator etiológico, logo após as internações por doenças respiratórias (23.778) e algumas doenças infecciosas e parasitárias (7.659) (DATASUS).

\subsubsection{CID 10 x Faixa Etária}

$\mathrm{Na}$ faixa etária compreendida entre um a quatro anos as internações por quedas foram as mais prevalentes correspondendo à $37,16 \%$ das causas externas. Na faixa etária compreendida entre cinco a nove anos as quedas também foram as maiores causas de internações, representando 47,73\% delas. A comparação entre as duas faixas etárias, no entanto, demonstrou uma clara diferença no tipo de acidentes sofridos. Entre um a quatro anos os envenenamentos; exposição ao fogo, fumaça, chamas e queimaduras; obstrução das vias aéreas por corpos estranhos (alimentos ou objetos); e afogamentos apresentaram-se mais relevantes (Gráfico 1). Essa diferença foi estatisticamente significante ( $<<0,0001)$. Já entre cinco e nove anos as quedas; exposição a forças mecânicas; os atropelamentos; acidentes de trânsito; contato com animais e plantas venenosos; exposição à corrente elétrica e acidentes de bicicleta foram as mais relevantes, como pode ser visto no Gráfico 1. Essa diferença também foi estatisticamente significante $(\mathrm{p}=0,0021)$.

Gráfico 1 - Morbidade Hospitalar por Causas Externas e Faixa Etária, Distrito Federal, 2015-2019.

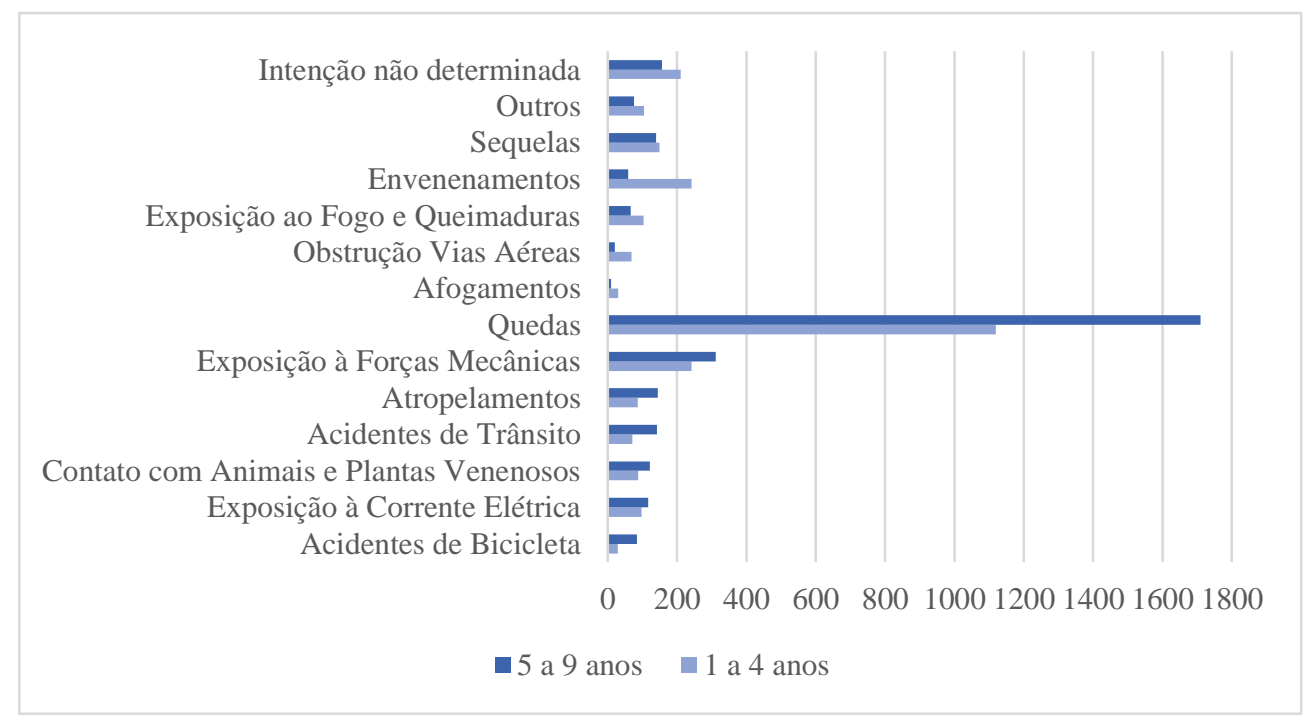

Fonte: Ministério da Saúde - Sistema de Informações Hospitalares do SUS (SIH/SUS)

Observar no Gráfico 1 a discrepância da quantidade de quedas em ambas as faixas etárias em relação aos demais acidentes. Os tipos de causas externas que aparecem no gráfico acima das quedas são de maioria ocorrida nas crianças de um a quatro anos, enquanto na parte inferior as ocorridas em maior parte nas crianças de cinco a nove anos. 
O cálculo das razões de riscos (Odds Ratio) demonstrou que crianças de um a quatro anos apresentam riscos significativamente aumentados de sofrerem em especial envenenamentos, obstrução de vias aéreas, afogamentos e queimaduras em comparação com os mesmos riscos em crianças de cinco a nove anos, como pode ser visto na Tabela 1. O cálculo das razões de riscos para cinco a nove anos em comparação com um a quatro anos mostrou que as crianças mais velhas apresentaram riscos mais significativos de sofrerem acidentes de bicicleta, seja colisão com outro veículo ou sem colisão, acidentes de trânsito, quedas e contato com animais peçonhentos e plantas venenosas (Tabela 1). Já os riscos relativos de exposição a forças mecânicas e à corrente elétrica em comparação nas duas faixas etárias não foram estatisticamente significantes, tendo em vista que os valores foram próximos de um (Tabela 1).

Tabela 1 - Razão dos riscos por tipos de acidentes e faixa etária, Distrito Federal, 2015-2019.

\begin{tabular}{lclc} 
Tipos de Acidentes & $\begin{array}{l}\text { Razão de Riscos } \\
\mathbf{1} \mathbf{4} 4 \text { anos X 5 a 9 anos }\end{array}$ & Tipos de Acidentes & $\begin{array}{l}\text { Razão de Riscos } \\
\mathbf{5} \text { a 9 anos X 1 a 4 anos }\end{array}$ \\
\hline Envenenamentos & 4.97 & Acidentes de bicicleta & 2.38 \\
Obstrução de Vias Aéreas & 3.92 & Acidentes de trânsito & 1.65 \\
Afogamentos & 3.64 & Atropelamentos & 1.36 \\
& & Quedas & 1.25 \\
& & Contato com Animais e & 1.15 \\
Exposição ao Fogo, Fumaça, & 1.89 & Plantas Venenosos & 1.05 \\
Chamas e Queimaduras & & Exposição à Forças & 0.98 \\
& Mecânicas \\
\hline
\end{tabular}

Fonte: Ministério da Saúde - Sistema de Informações Hospitalares do SUS (SIH/SUS)

Destaca-se na Tabela 1 a diferença dos riscos relativos nas duas faixas etárias e os principais tipos de acidente as quais cada uma dela está mais sujeita.

\subsubsection{CID $10 \times$ Sexo}

A comparação entre a ocorrência de causas externas e os sexos (masculino e feminino) demonstrou uma diferença estatisticamente relevante $(\mathrm{p}<0,0001)$ para todos os tipos de acidentes, com maior prevalência do sexo masculino. Além disso, no sexo masculino, as maiores causas de acidentes foram as quedas. A exposição à forças mecânicas animadas e inanimadas (lesões perfuro-cortantes, empalamento de objetos e mordeduras de animais), envenenamentos, exposição à correntes elétricas e atropelamentos também apareceram, porém em menor número. Enquanto as meninas os acidentes que mais sofreram foram, além das quedas em quantidade mais expressiva, exposição à forças mecânicas e envenenamentos, acidentes de trânsito e contato com animais peçonhentos e plantas venenosas (Tabela 2). Em ambos os sexos as causas de intenção não determinadas, sequelas de outras causas externas e as outras causas externas (não especificadas e não classificadas) ocuparam os segundos, terceiros e quintos lugares de internações (Tabela 2). 
Tabela 2 - Morbidade Hospitalar por Causas Externas e Sexo, Distrito Federal, 2015- 2019.

\begin{tabular}{|c|c|c|c|c|}
\hline \multirow{2}{*}{ Causas externas } & \multicolumn{2}{|c|}{ Sexo masculino } & \multicolumn{2}{|c|}{ Sexo feminino } \\
\hline & $\mathbf{N}$ & $\%$ & $\mathbf{N}$ & $\%$ \\
\hline Quedas & 1789 & $44,19 \%$ & 1041 & $40,84 \%$ \\
\hline Intenção Não Determinada & 390 & $9,63 \%$ & 223 & $8,75 \%$ \\
\hline Sequelas de outras causas externas & 360 & $8,89 \%$ & 272 & $10,67 \%$ \\
\hline Exposição à Forças Mecânicas & 330 & $8,15 \%$ & 223 & $8,75 \%$ \\
\hline $\begin{array}{l}\text { Outros (Não Especificados e Não } \\
\text { Classificados) }\end{array}$ & 236 & $5,83 \%$ & 152 & $5,96 \%$ \\
\hline Envenenamentos & 155 & $3,83 \%$ & 146 & $5,73 \%$ \\
\hline Exposição à Corrente Elétrica & 142 & $3,51 \%$ & 73 & $2,86 \%$ \\
\hline Atropelamentos & 141 & $3,48 \%$ & 90 & $3,53 \%$ \\
\hline Acidentes de Trânsito & 116 & $2,87 \%$ & 97 & $3,80 \%$ \\
\hline $\begin{array}{l}\text { Contato com Animais e Plantas } \\
\text { Venenosos }\end{array}$ & 116 & $2,87 \%$ & 94 & $3,69 \%$ \\
\hline Exposição ao Fogo e Queimaduras & 101 & $2,50 \%$ & 68 & $2,67 \%$ \\
\hline Acidentes de Bicicleta & 82 & $2,03 \%$ & 31 & $1,22 \%$ \\
\hline Obstrução de Vias Aéreas & 64 & $1,58 \%$ & 25 & $0,98 \%$ \\
\hline Afogamentos & 26 & $0,64 \%$ & 14 & $0,55 \%$ \\
\hline TOTAL & 4048 & $100 \%$ & 2549 & $100 \%$ \\
\hline
\end{tabular}

Fonte: Ministério da Saúde - Sistema de Informações Hospitalares do SUS (SIH/SUS)

Verifica-se na Tabela 2 que a comparação dos números absolutos dos acidentes nas duas faixas etárias mostra que todos ocorreram em maior número no sexo masculino. Observar que dentre os acidentes sofridos no sexo feminino, os envenenamentos, acidentes de trânsito e contato com animais e plantas venenosos foram mais expressivos que no sexo masculino.

\subsubsection{CID $10 \times$ Cor}

A análise por cor (branco, amarelo, preto, pardo, indígena e sem informação) das causas externas na faixa etária estudada mostrou que $82,52 \%$ das informações acerca desse dado não foram informadas. Os demais 17,48\% das causas foram compostos por: $12,89 \%$ pardos; $3,93 \%$ brancos; $0,49 \%$ pretos; $0,15 \%$ amarelos e $0,02 \%$ indígenas.

\subsubsection{CID 10 x Mês}

Os meses dos cinco anos consecutivos em que as causas externas ocorreram não foram estatisticamente relevantes ( $\mathrm{p}=0,6643$ ). Apesar disso, nos meses de janeiro, julho e dezembro, os acidentes ocorreram em maior número, fazendo com que esses se distanciassem mais da média de acidentes, como pode ser visto no Gráfico 2. Ademais, outros meses chegaram bem perto da quantidade de incidentes do mês de julho, como foi o caso de agosto, setembro e outubro. Os demais meses com menos incidentes apresentaram homogeneidade no número dessas ocorrências (Gráfico 2). 
Gráfico 2 - Distribuição Causas Externas por Meses, Distrito Federal, 2015-2019.

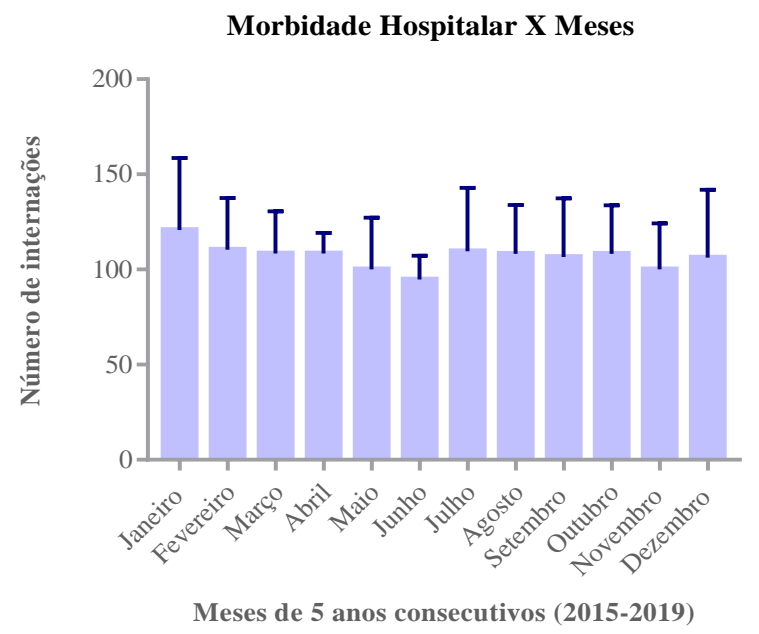

Fonte: Ministério da Saúde - Sistema de Informações Hospitalares do SUS (SIH/SUS)

Observar no Gráfico 2 que as barras mais espessas representam a média de casos em cada mês e as linhas o desvio padrão. A distribuição das médias ao longo dos meses é quase homogênea, destacando-se o desvio padrão dos meses de janeiro, julho e dezembro.

\subsubsection{CID 10 X Ano}

Como mostra o Gráfico 3, a análise da regressão não linear demonstrou uma tendência crescente das causas externas em relação aos anos (2015 a 2019).

Gráfico 3 - Distribuição Causas Externas por Anos, Distrito Federal, 2015-2019.

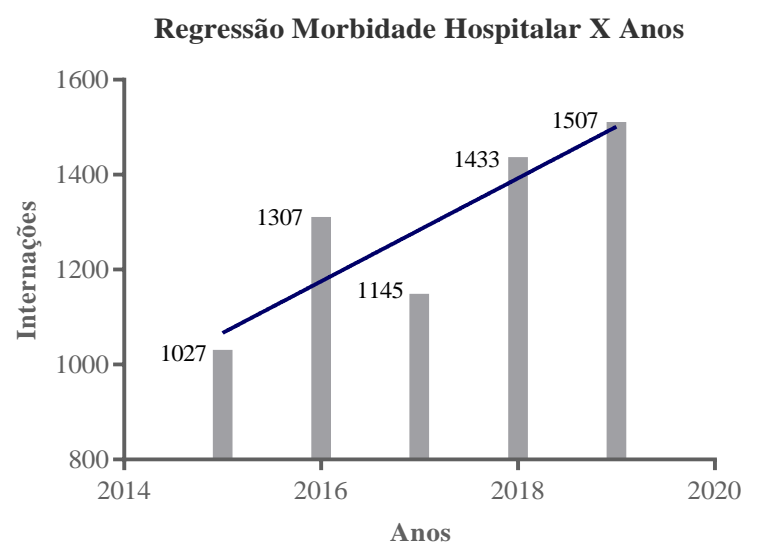

Fonte: Ministério da Saúde - Sistema de Informações Hospitalares do SUS (SIH/SUS)

Observar no Gráfico 3 que a linha diagonal representa a tendência dos acidentes ao longo do tempo. 


\subsection{Mortalidade}

No período estudado (2015 a 2019) no DF ocorreram 147 óbitos de crianças com idade entre um e nove anos por causas externas. Os dados reportados na mortalidade não necessariamente refletem os óbitos em relação à morbidade hospitalar descritos acima, a julgar que alguns dos óbitos podem ter ocorrido antes da internação hospitalar.

\subsubsection{Mortalidade X Faixa Etária}

A mortalidade por causas externas nesse período revelou que 37,99\% das crianças de um a quatro anos morreram por afogamento; 27,27\% em acidentes de trânsito e 6,82\% atropeladas, conforme mostra a Tabela 3. As principais causas de morte nas crianças de cinco a nove anos também foram as mesmas, apesar de ocuparem posições diferentes (Tabela 3).

Tabela 3 - Mortalidade por Causas Externas e Faixa Etária, DF, 2015-2019.

\begin{tabular}{|c|c|c|c|c|c|}
\hline \multirow{2}{*}{\multicolumn{2}{|c|}{ Causas externas }} & \multicolumn{2}{|c|}{1 a 4 anos } & \multicolumn{2}{|c|}{5 a 9 anos } \\
\hline & & $\mathbf{N}$ & $\%$ & $\mathbf{N}$ & $\%$ \\
\hline & Afogamentos & 35 & $39,77 \%$ & 7 & $11,87 \%$ \\
\hline & Acidentes de Trânsito & 24 & $27,27 \%$ & 28 & $47,46 \%$ \\
\hline & Atropelamentos & 6 & $6,82 \%$ & 9 & $15,26 \%$ \\
\hline & Obstrução de Vias Aéreas & 5 & $5,68 \%$ & 2 & $3,39 \%$ \\
\hline & Quedas & 3 & $3,41 \%$ & 1 & $1,69 \%$ \\
\hline & Exposição à Forças Mecânicas & 3 & $3,41 \%$ & 3 & $5,09 \%$ \\
\hline & Sequelas de outras causas externas & 3 & $3,41 \%$ & 0 & $0,00 \%$ \\
\hline & Exposição ao Fogo e Queimaduras & 2 & $2,27 \%$ & 1 & $1,69 \%$ \\
\hline & $\begin{array}{l}\text { Contato com Animais e Plantas } \\
\text { Venenosos }\end{array}$ & 2 & $2,27 \%$ & 0 & $0,00 \%$ \\
\hline & Envenenamentos & 2 & $2,27 \%$ & 1 & $1,69 \%$ \\
\hline & Exposição à Corrente Elétrica & 1 & $1,14 \%$ & 1 & $1,69 \%$ \\
\hline & Acidentes de Bicicleta & 1 & $1,14 \%$ & 0 & $0,00 \%$ \\
\hline & Intenção Não Determinada & 1 & $1,14 \%$ & 5 & $8,48 \%$ \\
\hline & Outros & 0 & $0,00 \%$ & 1 & $1,69 \%$ \\
\hline & TOTAL & 88 & $100 \%$ & 59 & $100 \%$ \\
\hline
\end{tabular}

Ministério da Saúde - Sistema de Informações Hospitalares do SUS (SIH/SUS)

Observa-se na Tabela 3 que nas crianças de um a quatro anos os afogamentos constituíram a maior parte das causas de mortalidade, enquanto na faixa etária de cinco a nove anos os acidentes de trânsito.

\section{Discussão}

Assim como os estudos presentes na literatura apresentam, as quedas continuam sendo o principal tipo de acidente sofrido pela população pediátrica que leva às internações hospitalares. (Batalha et al., 2016; Degeorge, 2020; Olsen \& Kennedy, 2020; Simas \& Souza, 2019). As quedas de mesma altura fazem parte do desenvolvimento natural da coordenação motora (Kramlich \& Dende, 2016), no entanto, existem fatores protetores associados à redução das quedas tanto do mesmo nível quanto de outros mais altos e, portanto, mais graves (como por exemplo do leito, de cadeiras, de mobílias, de playgrounds e árvores). 
Como medidas protetivas, portanto, podem ser destacadas: reduzir os desníveis nos pisos, utilizar barreiras como portões de segurança nas escadas, proteções nas janelas e grades de proteção nas camas, supervisionar as crianças em escadas, entre outros (Haarbauer-Krupa et al., 2019). Há ainda outros fatores associados à redução das lesões ocorridas nas quedas, como por exemplo brinquedos de playground mais baixos e uso de superfícies amortecedoras embaixo destes (Olsen \& Kennedy, 2020).

Os riscos relativos encontrados no estudo vão ao encontro da literatura. As intoxicações aparecem como de maiores riscos para crianças menores de 4 anos tendo em vista que ainda não possuem uma capacidade discriminativa aguçada e, portanto, podem confundir por exemplo medicamentos com doces, ou ainda os frascos de produtos de limpeza com frascos de bebidas, devido às cores e formatos parecidos (Plevová \& Nedělová, 2018). A prevenção desse tipo de incidente, portanto, pode ser feita por meio do armazenamento adequado de medicamentos e produtos de limpeza, maior supervisão dos responsáveis, ensino adequado às crianças, evitando comparações de medicamentos com balas e doces e utilização de embalagens que impeçam a abertura por crianças (Amorim et al., 2017; Anderson et al., 2016).

Além disso, devido à curiosidade e maior aquisição das habilidades motoras finas levam frequentemente objetos à boca, que são acidentalmente aspirados, obstruindo as vias aéreas. Outros fatores como dentição incompleta, coordenação da mastigação e deglutição imatura e vias aéreas de menor diâmetro apresentam riscos aumentados de crianças de 1 a 4 anos sofrerem obstruções de vias aéreas (Lluna et al., 2016; Ministero della Salute, 2017; Simas \& Souza, 2019). Nesse cenário, além da constante vigilância e acompanhamento dos responsáveis também durante a alimentação, é importante que estes saibam como realizar corretamente a manobra de Heimilch nesses casos, visto que é uma situação potencialmente fatal.

A constatação dos afogamentos na faixa etária de 1 a 4 anos como mais prevalente também é concordante com a literatura (Clemens et al., 2016; Leavy et al., 2016; Liu et al., 2019). Além das características já apontadas da curiosidade e imaturidade cognitiva para prever as situações de perigo, outro fator que predispõe crianças nessa faixa etária a se afogarem é a desproporção anatômica entre a cabeça e o restante do corpo (Filocomo et al., 2017; Gonçalves et al., 2019). Sendo mais pesada, facilmente podem favorecer a queda em piscinas ou dificultar a emersão da criança ao cair em baldes ou bacias em banhos, por exemplo.

Em relação aos acidentes de transporte (bicicleta, trânsito e atropelamentos) apareceram em maior número nas crianças de 5 a 9 anos, como mostra a literatura (Cunha \& Godoy, 2017; Doud et al., 2016). Esses dados podem ser explicados pelo fato de que crianças maiores possuem maior independência, além de nessa fase começarem a vida escolar, e, portanto, serem transportadas em veículos com maior frequência e serem expostas à situações de risco maiores, como atravessar faixas de pedestres sem um responsável adulto.

A preponderância do sexo masculino nos acidentes também já é bem descrita na literatura (Batalha et al., 2016; Degeorge, 2020; Malta et al., 2016) e esse fator e os tipos de acidentes mais sofridos por esse sexo podem ser justificados pelas atividades mais realizadas pelos meninos, que mais frequentemente envolvem velocidade, força, impacto corporal e, assim ficam expostos a maiores riscos (Filocomo et al., 2017; Montana et al., 2020).

A grande quantidade das causas externas de intenções não determinadas, sequelas de outras causas externas e as não determinadas e não classificadas constituem um problema, pois o diagnóstico e compreensão integral desses incidentes é fundamental para que seja realizada uma boa prevenção em saúde.

Em relação à ocorrência de causas externas na pediatria e a cor da pele, os estudos apontam a preponderância da cor preta (Degeorge, 2020; Privette et al., 2018; Steinbach et al., 2016). Não obstante, esse estudo mostrou uma taxa de 0,49\% de acidentes na população negra dessa faixa etária. Tais dados podem refletir a realidade da declaração de cor no Brasil, tendo em vista que a maior parte desta se declara parda (46,8\%) e uma parcela preta (9,4\%), segundo a PNAD 2019 (Instituto Brasileiro de Geografia e Estatística [IBGE], 2020). Já a porcentagem de dados não informados e o preenchimento incorreto dos dados 
consiste numa problemática, tendo em vista que impossibilita uma análise real da situação da ocorrência de causas externas nessa faixa etária e sua relação com fatores sociodemográficos e, portanto, impossibilita a atuação nessa correlação.

A literatura apresenta que a maioria dos acidentes ocorrem no verão e/ou época de férias, nos meses de dezembro, janeiro, junho e julho (Brito et al., 2016; Chauvin et al., 2019; Cunha \& Godoy, 2017), porém o presente estudo não encontrou uma correlação estatística significativa entre a ocorrência dessas causas externas com os 12 meses do ano. Apesar dos meses de janeiro, julho e dezembro terem apresentado um número maior de casos, o fato de agosto, setembro e outubro terem ficado próximo à essa quantidade e os demais apresentarem comportamento bem distribuído tem como hipótese a falta de estações do ano bem definidas no Distrito Federal e a não variação significativa das temperaturas ao longo dos meses. Outra hipótese é de que as férias escolares podem coincidir com os períodos de férias dos responsáveis e, por consequência, aumentar a supervisão dentro de casa, ou ainda que os acidentes podem ocorrer em outros locais além do domicílio e, portanto, maior tempo em casa não representaria necessariamente maior exposição à riscos. As informações do local de ocorrência e se a criança estava sob supervisão se mostram essenciais para a análise do perfil de ocorrência dessas causas externas e assim a atuação na prevenção, no entanto, ela não pode ser analisada, pois os dados disponibilizados não informavam nenhuma das duas variáveis.

A tendência crescente de ocorrência das causas externas nessa faixa etária é uma preocupação, tendo em vista que acarretam em diversos tipos de custos, tanto para as crianças quanto para suas famílias e sociedade (Malta et al., 2016). Isso porque além dos sofrimentos iniciais trazidos pelos acidentes, as crianças podem ainda sofrer com as sequelas desses acidentes, incluindo limitações físicas, dores crônicas e problemas psicológicos (Mitchell et al., 2018; Mukama et al., 2019). Além disso, as famílias dessas crianças hospitalizadas, em especial as que apresentam estados de saúde mais graves, experenciam medo e ansiedade ao verem o sofrimento e as mudanças de aparência e comportamento de seus filhos (Foster et al., 2016). Há ainda os custos dessas hospitalizações e prejuízos para o Estado, que poderiam ser melhor investidos na prevenção. Essa tendência reforça a importância de os profissionais de saúde orientarem os cuidadores acerca do desenvolvimento psicomotor da criança, de acordo com a faixa etária, a fim de que promovam um ambiente protetivo na infância.

A análise da mortalidade mostra que os afogamentos continuam, portanto, constituindo um grave risco à vida das crianças, em especial de 1 a 4 anos, tendo em vista que são incidentes silenciosos e que podem rapidamente levar à óbito (Loux et al., 2021; Ribeiro et al., 2019). Um estudo apontou inclusive um caso de afogamento com apenas 30 segundos sem supervisão e em $5 \mathrm{~cm}$ de profundidade de água corroborando para a conscientização da importância da supervisão dos responsáveis em todos os momentos (Peden et al., 2018).

Os acidentes de trânsito e atropelamentos também apresentaram maiores taxas de mortalidade e isso pode ser explicado pelo fato de serem acidentes, no geral, mais graves que os demais, pela cinemática do trauma. Tendo em vista que as crianças devem ser corretamente transportadas nos veículos, quando isso não ocorre, o risco dessa criança sofrer consequências graves é grande. Esses dados mostram a importância do transporte em dispositivos adequados para a idade e peso das crianças, além da prevenção desses acidentes por meio da educação no trânsito. Também na cinemática do trauma dos atropelamentos, devido à altura das crianças, no geral as partes do corpo mais atingidas são a cabeça e o pescoço e múltiplos órgãos, causando, portanto, danos mais severos e com maiores chances de óbitos (Cunha \& Godoy, 2017; Pinto et al., 2016).

\subsection{Limitações do Estudo}

Tendo em vista que se tratou de uma análise com fontes de informações secundárias, uma das limitações do estudo é a falta de informações acerca de algumas variáveis, como: cor de pele, local de ocorrência do acidente e maior especificação da etiologia dos não classificados. Por esse motivo reforça-se a importância do preenchimento correto e completo das fichas de notificações para seja possível analisar e compreender melhor a situação das crianças expostas à diferentes riscos de sofrerem 
acidentes. Sugere-se ainda mais estudos para analisar a relação das causas externas com os meses do ano e outros fatores que podem influenciar a sua ocorrência bem distribuída.

\subsection{Contribuições para a Área da Saúde Pública}

O estudo confirma o perfil das internações hospitalares e óbitos por causas externas na infância que vem sendo apresentados na literatura e esclarece alguns dos aspectos de ocorrência dessas causas no Distrito Federal. Evidencia ainda o caráter da evitabilidade desses eventos e, portanto, demonstra ser indispensável o envolvimento das famílias, sociedade, governos e primordialmente equipes de saúde na prevenção das causas externas na infância, assegurando o direito das crianças de se desenvolverem integralmente em ambientes seguros.

\section{Conclusões}

Os principais resultados do estudo mostram que o sexo masculino continua constituindo a maioria das crianças acidentadas e, portanto, deve-se continuar enfatizando a prevenção destes acontecimentos nessa população. Concluiu ainda que os acidentes ocorridos nas duas faixas etárias têm perfis diferentes e, assim, é necessário entender esses diferentes tipos para atuar com mais propriedade na prevenção desses incidentes. Outra conclusão do estudo foi o perigo dos afogamentos, atropelamentos e acidentes de trânsito resultarem mais frequentemente em óbitos. Assim, considerando que os profissionais de saúde são os principais responsáveis pela educação em saúde dos responsáveis, devem reforçar a importância da supervisão e promoção de ambientes seguros para as crianças, bem como estimular a atuação conjunta com a sociedade e as esferas envolvidas na prevenção dos acidentes na infância- educação, trânsito, planejamento urbano, entre outros.

\section{Referências}

Amorim, M. L. P., Mello, M. J. G, \& Siqueira, M. T. (2017). Poisoning in children and adolescents notified at a toxicology center in the Northeast of Brazil. Revista Brasileira de Saúde Materno Infantil, 17: 765-772. https://doi: 10.1590/1806-93042017000400009.

Anderson, M., Hawkins, L., Eddleston, M., Thompson, J. P., Vale, J. A., \& Thomas, S. H. L. (2016). Severe and fatal pharmaceutical poisoning in young children in the UK. Archives of Disease in Childhood, 101 (7): 653-656. https://doi: 10.1136/archdischild-2015-309921.

Batalha, S., Salva, I., Santos, J., Albuquerque, C., Cunha, F. \& Sousa, H. (2016). Acidentes em Crianças e Jovens, Que Contexto e Que Abordagem: Experiência de Nove Meses no Serviço de Urgência num Hospital de Nível II. Acta Pediátrica Portuguesa, 47:30-37. https://doi: 10.25754/pjp.2016.6207.

Brito, J. G., Pedroso, B. R. P., \& Martins, C. B. G. (2016). Acidentes domiciliares por forças mecânicas inanimadas em crianças, adolescentes e jovens. Texto \& Contexto Enfermagem, 25(2) :e4180014. https://doi: 10.1590/0104-07072016004180014.

Brito, M. A., Melo, A. M. N., Veras, I. C, Oliveira, C. M. S., Bezerra, M. A. R., \& Rocha, S. S. (2017). Fatores de risco no ambiente doméstico para quedas em crianças menores de cinco anos. Revista Gaúcha de Enfermagem, 38(3). https://doi: 10.1590/1983-1447.2017.03.2017-0001.

Chang, S. S. M., Symons, R., \& Ozanne-Smith, J. (2018). Child road traffic injury mortality in Victoria, Australia (0-14 years), the need for targeted action. Injury, 49(3): 604-612. https://doi: 10.1016/j.injury.2017.12.018.

Chauvin, M., Kosatsky, T., Bilodeau-Bertrand, M., Gamache, P., Smargiassi, A., \& Auger, N. (2019). Hot weather and risk of drowning in children: Opportunity for prevention. Preventive Medicine, 130: 105885- 105886. https://doi: 10.1016/j.ypmed.2019.105885.

Clemens, T., Tamim, H., Rotondi, M., \& Macpherson, A. K. (2016). A population based study of drowning in Canada. BMC Public Health, 16. https://doi: $10.1186 / \mathrm{s} 12889-016-3221-8$.

Companhia de Planejamento do Distrito Federal. (2018). Retratos Sociais DF 2018: A população infantil no Distrito Federal. https://www.codeplan.df.gov.br/wp-content/uploads/2020/05/Estudo-Retratos-Sociais-DF-2018-A-popula\%C3\%A7\%C3\%A3o-infantil-no-DistritoFederal.pdf

Companhia de Planejamento do Distrito Federal. (2020). Atlas do Distrito Federal 2020. https://www.codeplan.df.gov.br/atlas-do-distrito-federal-2020.

Cunha, S. M. P., \& Godoy, C. B. (2017). Transportation accidents among children, adolescents and young people: epidemiological study Acidentes de transporte terrestre entre crianças, adolescentes e jovens: estudo epidemiológico. Revista de Pesquisa: cuidado é fundamental Online 9(4): 1021-1027. https://doi: 10.9789/2175-5361.2017.v9i4.1021-1027.

Degeorge, K. C., Neltner, C. A., \& Neltner, B. J. (2020) Prevention of Unintentional Childhood Injury. American Family Physician, 102 (7): $411-417$. https://www.aafp.org/afp/2020/1001/p411.html. 
Doud, A. N., Weaver, A. A., Talton, J. W., Barnard, R. T., Petty, J., \& Stitzel, J. D. (2016). Evaluation of developmental metrics for utilization in a pediatric advanced automatic crash notification algorithm. Traffic Injury Prevention, 17(1): 65-72. https://doi:10.1080/15389588.2015.1051223.

Ehrhardt, J., Xu, Y., Khoury, J., Yolton, K., Lanphear, B., \& Phelan, K. (2017). Cognitive and motor abilities of young children and risk of injuries in the home. Injury Prevention, 23(1): 16-21. https://doi: 10.1136/injuryprev-2016-042031.

Filocomo, F. R. F., Harada, M. J. C. S, Mantovani, R., \& Ohara, C. V. S. (2017). Perfil dos acidentes na infância e adolescência atendidos em um hospital público. Acta Paulista de enfermagem, 30(3): 287-294. https://doi: 10.1590/1982-0194201700044.

Foster, K., Young, A., Mitchell, R., Van, C., \& Curtis, K. (2017). Experiences and needs of parents of critically injured children during the acute hospital phase: A qualitative investigation. Injury, 48(1): 114-120. https://doi:10.1016/j.injury.2016.09.034.

Gonçalves, A. C., Araújo, M. P. B., Paiva, K. V., Menezes, C. S. A., Silva, A. E. M. C., Santana, G. O., Ortolan, E. V. P., \& Lourenção, P. L. T. A. (2019). Acidentes na infância: casuística de um serviço terciário em uma cidade de médio porte do Brasil. Revista do Colégio Brasileiro de Cirurgiões, 46 (2): e2104 . https://doi: 10.1590/0100-6991e-20192104.

Goniewicz, K., Goniewicz, M., Pawłowski, W., \& Fiedor, P. (2018). Management of child injuries in traffic and other accidents: the WHO policy guidelines. Health Problems of Civilization, 12 (3): 157-162. https://doi: 10.5114/hpc.2018.76516.

Gurgel, A. K. C., \& Monteiro, A. I. (2016). Prevenção de acidentes domésticos infantis: susceptibilidade percebida pelas cuidadoras. Revista de Pesquisa Cuidado é Fundamental Online, 8(4): 5126-5135. https://doi: 10.9789/2175-5361.2016.v8i4.5126-5135.

Haarbauer-Krupa, J., Haileyesus, T., Gilchrist, J., Mack, K. A., Law, C. S., \& Joseph, A. (2019). Fall-related traumatic brain injury in children ages 0-4 years. Journal of Safety Research, 70: 127-133. https://doi: 10.1016/j.jsr.2019.06.003.

Instituto Brasileiro de Geografia e Estatística. (2020). Características gerais dos domicílios e dos moradores 2019 , PNAD 2019 Contínua. https://biblioteca.ibge.gov.br/visualizacao/livros/liv101707_informativo.pdf.

Khazaei, S., Mazharmanesh, S., Khazaei, Z., Goodarzi, E., Mirmoini, R., Mohammadian-Hafshejani, A., \& Khazaei, S. (2016). An epidemiological study on the incidence of accidents in the Hamadan province during 2009 to 2014. Pajouhan Scientific Journal, 14(2). https://psj.umsha.ac.ir/article-1-169-en.html.

Kramlich, D.L., \& Dende, D. B. T. (2016). Development of a pediatric fall risk and injury reduction program. Pediatric Nursing, 42 (2): 77-82. https://GALE: A491256105

Leavy, J. E., Crawford, G., Leaversuch, F., Nimmo, L., McCausland, K., \& Jancey, J. (2016). A Review of Drowning Prevention Interventions for Children and Young People in High, Low and Middle Income Countries. Journal of Community Health, 41, (2): 424-441. https://doi: 10.1007/s10900-015-0105-2.

Lima-Costa, M. F., \& Barreto, S. M. (2003). Tipos de estudos epidemiológicos: conceitos básicos e aplicações na área do envelhecimento. Epidemiologia e Serviços de Saúde, 12(4): 189-201. https://doi: 10.5123/S1679-49742003000400003.

Liu, Z., Kong, F., Yin, L., Wang, A., Xiong, L., \& Xie, D. (2019). Epidemiological characteristics and influencing factors of fatal drowning in children under 5 years old in Hunan Province, China: case-control study. BMC Public Health, 19 (1): 1. https://doi: 10.1186/s12889-019-7241-z.

Lluna, J., Olabarri, M., Domènech, A., Rubio, B., Yagüe, F., Benítez, M. T.,Esparza, M. J., \& Mintegi, S. (2016). Recomendaciones sobre la prevención de aspiraciones de cuerpos extraños. Anales de Pediatria, 86. https://doi: 10.1016/j.anpedi.2016.04.013.

Loux, T., Mansuri, F., Brooks, S. E., Slye, N., Lewis, B., Lu, Y.,Chen, H., \& Kip, K. E. (2021). Factors associated with pediatric drowning admissions and outcomes at a trauma center, 2010-2017. American Journal of Emergency Medicine, 39: 86-91. https://doi: 10.1016/j.ajem.2020.01.025.

Mackay, J. M., \& Ryan, M. A. (2018). Human rights-based approach to unintentional injury prevention. Injury Prevention, 24(1): i67--i73. https://doi: 10.1136/injuryprev-2017-042692.

Malta, D. C., Mascarenhas, M. D. M., Silva, M. M. A., Carvalho, M. G. O., Barufaldi, L. A., Avanci, J. Q., \& Bernal, R. T. I. (2016). A ocorrência de causas externas na infância em serviços de urgência: aspectos epidemiológicos, Brasil, 2014. Ciência \& Saúde Coletiva, 21: 3729-3744. https://doi: 10.1590/1413812320152112.17532016

Ministério da Saúde. (2015). Saúde Brasil 2014: uma análise da situação de saúde e das causas externas. Secretaria de Vigilância em Saúde, Departamento de Vigilância de Doenças e Agravos Não Transmissíveis e Promoção da Saúde. http://bvsms.saude.gov.br/bvs/p ublicacoes/saude_brasil_2014_analise_situacao.pdf.

Ministero della Salute. (2017). Linee di indirizzo per la prevenzione del soffocamento da cibo, Italia. http://www.salute.gov.it/imgs/C_17_pubblicazioni_2618_allegato.pdf.

Mitchell, R. J, Curtis, K., \& Foster, K. (2018). A 10-year review of child injury hospitalisations, health outcomes and treatment costs in Australia. Injury Prevention, 24(5): 344-350. https://doi: 10.1136/injuryprev-2017-042451.

Montana, A., Salerno, M., Feola, A., Asmundo, A., Di Nunno, N., Casella, F., Manno, E., Colosimo, F., Serra, R., \& Di Mizio, G. (2020). Risk Management and Recommendations for the Prevention of Fatal Foreign Body Aspiration: Four Cases Aged 1.5 to 3 Years and Mini-Review of the Literature. International Journal of Environmental of Research and Public Health, 17(13):1. https://doi:10.3390/ijerph17134700.

Mukama, T., Ssemugabo, C., Halage, A. A, Gibsn, D. G., Paichadze, N., \& Ndejjo, R. (2019). Costs of unintentional injuries among children in an urban slum community in Kampala city, Uganda. International journal of injury control and safety promotion, 26 (2): 129-136. https://doi: $10.1080 / 17457300.2018 .1515228$.

Olsen, H., \& Kennedy, E. (2020). Safety of School Playgrounds: Field Analysis From a Randomized Sample. Journal of School Nursing, 36(5) :369-375. https://doi: $10.1177 / 1059840519827364$. 
Research, Society and Development, v. 10, n. 10, e202101018725, 2021

(CC BY 4.0) | ISSN 2525-3409 | DOI: http://dx.doi.org/10.33448/rsd-v10i10.18725

Olsen, L. L., Ishikawa, T., Mâsse, L. C, Chan, G., \& Brussoni, M. (2018). Risk Engagement and Protection Survey (REPS): developing and validating a survey tool on father attitudes towards child injury protection and risk engagement. Injury Prevention, 24(2): 106-112. https://doi.org/10.1136/injuryprev-2017-042413.

Organização Mundial da Saúde. (1975). Classificação estatística internacional de doenças: manual de lesões e causas de óbito 9. Centro Brasileiro Classificação Doenças em Português, 1979.

Peden, A. E., Franklin, R. C, \& Pearn, J. H. (2018). Unintentional fatal child drowning in the bath: A 12-year Australian review (2002-2014). Journal of Paediatris and Child Health, 54(2): 153-159. https://doi: 10.1111/jpc.13688.

Pinto, L. W., Ribeiro, A. P., Bahia, C. A., \& Freitas, M. G. (2016). Urgent and emergency care for pedestrians injured in Brazilian traffic. Ciências e Saúde Coletiva, 21(12): 3673-3682. https://doi: 10.1590/1413-812320152112.17722016.

Plevová, I., \& Nedělová, M. (2018). Safe storage of household substances to prevent child poisoning. Central European Journal of Nursing and Midwifery, 9. https://doi: 10.15452/CEJNM.2018.09.0014.

Privette, F., Nwosu, A., Pope, C. N., Yang, J., Pressley, J. C. (2018). Factors Associated With Child Restraint Use in Motor Vehicle Crashes. Clinical Pediatrics, 57(12): 1423-1431. https://doi:10.1177/0009922818786002.

Resolução $\mathrm{n}^{\mathrm{o}}$ 466, de 12 de dezembro de 2012. Conselho Nacional de Saúde, Ministério da Saúde, Brasil, 2012. https://bvsms.saude.gov.br/bvs/saudelegis/cns/2013/res0466_12_12_2012.html.

Ribeiro, A., Barros, M., Pereira, I. A., Lírio, C., Pais, I. P., \& Couto, M. L. (2019). Conhecimentos e práticas parentais sobre medidas preventivas de acidentes domésticos e de viação. Revista Portuguesa de Medicina Geral e Familiar, 35(3): 186-195. https://doi: 10.32385/rpmgf.v35i3.12286.

Saadati, M., Tabrizi, J. S., Rezapour, R., \& Kalajahi, R. A. (2020). Home injury prevention attitude and performance: a community-based study in a designated safe community. Journal of injury \& violence research, 12 (2). https://doi: 10.5249/jivr.v12i2.1506.

Schwebel, D. C. (2019). Why "accidents" are not accidental: Using psychological science to understand and prevent unintentional child injuries. The American psychologist, 74(9), 1137-1147. https://doi: 10.1037/amp0000487.

Simas, V.F.,\& Souza, A.S. (2019). Perfil de crianças hospitalizadas na pediatria vítimas de acidentes na primeira infância. Revista Pró-UniverSUS, 10 (1): 2528. https://doi: 10.21727/rpu.v10i1.1633.

Sleet, D.A. (2018). The global challenge of child injury prevention. International Journal of Environmental Research and Public Health, 15(9). https://doi: 10.3390/ijerph15091921.

Steinbach, R., Green, J., Kenward, M. G., \& Edwards, P. (2016). Is ethnic density associated with risk of child pedestrian injury? A comparison of inter-census changes in ethnic populations and injury rates. Ethnictiy \& Health, 21(1): 1-19. https://doi: 10.1080/13557858.2014.985637.

Zonfrillo, M. R., Gittelman, M. A, Quinlan, K. P., \& Pomerantz, W.J. (2018). Outcomes after injury prevention counselling in a paediatric office setting: a 25year review. BMJ Paediatrics Open, 2 (1). https://doi: 10.1136/bmjpo-2018-000300. 